\title{
Phosphorylation by aurora kinase A induces Mdm2- mediated destabilization and inhibition of p53
}

\author{
Hiroshi Katayama ${ }^{1}$, Kaori Sasai ${ }^{1}$, Hidehiko Kawai ${ }^{2,3}$, Zhi-Min Yuan $^{2}$, Jolanta Bondaruk ${ }^{4}$, Fumio Suzuki ${ }^{3}$, \\ Satoshi Fujii ${ }^{1}$, Ralph B Arlinghaus ${ }^{1}$, Bogdan A Czerniak ${ }^{4} \&$ Subrata Sen $^{1}$
}

\begin{abstract}
Aurora kinase A (also called STK15 and BTAK) is overexpressed in many human cancers. Ectopic overexpression of aurora kinase A in mammalian cells induces centrosome amplification, chromosome instability and oncogenic transformation, a phenotype characteristic of loss-of-function mutations of p53. Here we show that aurora kinase A phosphorylates p53 at Ser315, leading to its ubiquitination by Mdm2 and proteolysis. p53 is not degraded in the presence of inactive aurora kinase A or ubiquitinationdefective Mdm2. Destabilization of p53 by aurora kinase A is abrogated in the presence of mutant Mdm2 that is unable to bind p53 and after repression of Mdm2 by RNA interference. Silencing of aurora kinase A results in less phosphorylation of p53 at Ser315, greater stability of p53 and cell-cycle arrest at G2-M. Cells depleted of aurora kinase A are more sensitive to cisplatininduced apoptosis, and elevated expression of aurora kinase A abolishes this response. In a sample of bladder tumors with wildtype p53, elevated expression of aurora kinase A was correlated with low p53 concentration. We conclude that aurora kinase A is a key regulatory component of the p53 pathway and that overexpression of aurora kinase A leads to increased degradation of p53, causing downregulation of checkpoint-response pathways and facilitating oncogenic transformation of cells.
\end{abstract}

Aurora kinase A is a member of a new family of serine/threonine kinases that includes Drosophila melanogaster Aurora and Saccharomyces cerevisiae Ipl1 kinase, both of which are essential for controlling normal chromosome segregation and centrosome functions ${ }^{1-3}$. Aurora kinase A has been implicated in regulating centrosome function, spindle assembly, spindle maintenance and mitotic commitment in cells ${ }^{4-7}$. AURKA, encoding aurora kinase $\mathrm{A}$, is a putative oncogene that is amplified and overexpressed in many human cancers $^{8-13}$. The molecular targets of aurora kinase A have not been well characterized. We previously reported that phosphorylationmediated feedback between aurora kinase A and protein phosphatase 1 operates through mitosis and that disruption of this interaction results in defects in chromosome segregation ${ }^{14}$.

Overexpression of aurora kinase $\mathrm{A}^{8}$ and loss of wild-type p53 function induce similar phenotypes of centrosome amplification and aneuploidy in cells ${ }^{15,16}$. These observations suggest that gain of aurora kinase A function and loss of wild-type p 53 function may be interdependent in common pathways. The finding that human tumors with elevated expression of aurora kinase A have wild-type TRP53 (encoding p53) also suggests that gain of aurora kinase A function may cause loss of wild-type p53 function, contributing to malignant transformation.

p53 induces growth arrest or apoptosis in cells exposed to stress and is frequently mutated or deleted in human cancers. Expression of p53 is controlled by Mdm2, which promotes ubiquitination by
E3 ubiquitin ligase activity and degradation of p53 by the cytoplasmic $26 \mathrm{~S}$ proteasome ${ }^{17}$. Stability and activity of p53 are also regulated by post-translational modifications ${ }^{18-23}$ including phosphorylation, acetylation, glycosylation and attachment of a small ubiquitin-related modifier protein. Phosphorylation at multiple sites is the predominant mechanism known to stabilize and abrogate Mdm2-mediated ubiquitination and activates p53. In contrast, phosphorylation of the core domain at Thr 155 by the COP9 signalosome has been reported to target p53 for degradation ${ }^{24}$. The present study investigated whether phosphorylation by aurora kinase A also regulates p53 activity.

\section{RESULTS}

Aurora kinase A phosphorylates and interacts with p53

We first investigated the ability of aurora kinase A to phosphorylate p53 in an in vitro kinase assay. We incubated bacterially expressed glutathione S-transferase (GST) and a GST-p53 fusion protein with aurora kinase A immunoprecipitated from mitotic HeLa cells and $\gamma^{32} \mathrm{P}$ ATP. The aurora kinase A immunocomplex clearly phosphorylated GST-p53 (Fig. 1a). To confirm the specificity of aurora kinase A in phosphorylating p53, we used immunoprecipitated wild-type and kinase-inactive aurora kinase A (K162R) in an in vitro kinase assay with GST-p53. Wild-type aurora kinase A phosphorylated p53 but the kinase-inactive mutant did not (Fig. 1b), confirming that aurora

${ }^{1}$ Department of Molecular Pathology, Division of Pathology \& Laboratory Medicine, University of Texas M.D. Anderson Cancer Center, Houston, Texas 77030, USA. ${ }^{2}$ Department of Cancer Cell Biology, Harvard School of Public Health, Boston, Massachusetts 02115, USA. ${ }^{3}$ Department of Molecular Radiobiology, Research Institute for Radiation Biology and Medicine, Hiroshima University, Hiroshima 734-8553, Japan. ${ }^{4}$ Department of Pathology, Division of Pathology \& Laboratory Medicine, University of Texas M.D. Anderson Cancer Center, Houston, Texas 77030, USA.Correspondence should be addressed to S.S. (ssen@mail.mdanderson.org). 
kinase A specifically phosphorylates p53 in vitro. We examined the pattern of phosphorylation of $\mathrm{p} 53$ by aurora kinase A in vitro by phosphorylated-amino-acid and two-dimensional phosphopetide analysis of ${ }^{32}$ P-labeled GST-p53 after digestion with N-tosyl-L-phenylalaninechloromethyl ketone-trypsin ${ }^{25}$. We detected radioactive signal at the phosphoserine marker, indicating that aurora kinase A phosphorylates p53 at a serine residue (Fig. 1c). Two-dimensional phosphopeptide analysis showed a phosphorylated peptide in the second dimension (Fig. 1d). Edman degradation ${ }^{26}$ showed that the highest radioactivity was released after the ninth cycle (Fig. 1e), corresponding to the serine at position 315 of p53. An in vitro kinase assay using GST-p53 showed that wild-type p53, but not S315A mutant p53, was phosphorylated by aurora kinase A (Fig. 1f).
To investigate phosphorylation of Ser315 by aurora kinase A in vivo, we expressed wild-type and S315A mutant p53 with either constitutively activated (T288D) or kinase-inactive mutants of aurora kinase A in HEK293 cells. We detected a strong signal with an antibody to p53 phosphorylated at Ser315 in cells expressing the constitutively activated aurora kinase $\mathrm{A}$ and weaker signals in the cells expressing the kinase-inactive control, probably due to endogenous aurora kinase A in these cells. We detected no signal in cells expressing S315A mutant p53, confirming that aurora kinase A phosphorylates p53 at Ser315 in vivo (Fig. 1g).

To determine whether the interaction between aurora kinase A and p53 occurs in vitro, we incubated ${ }^{35}$ S-labeled in vitro-translated p53 constructs expressing either GST-tagged full-length aurora kinase A or a

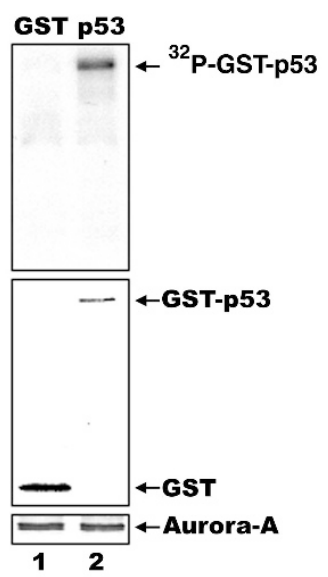

d

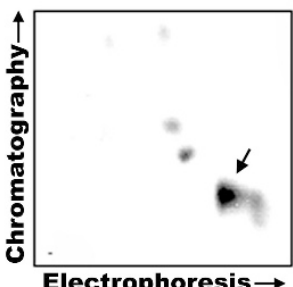

Electrophoresis $\rightarrow$

f

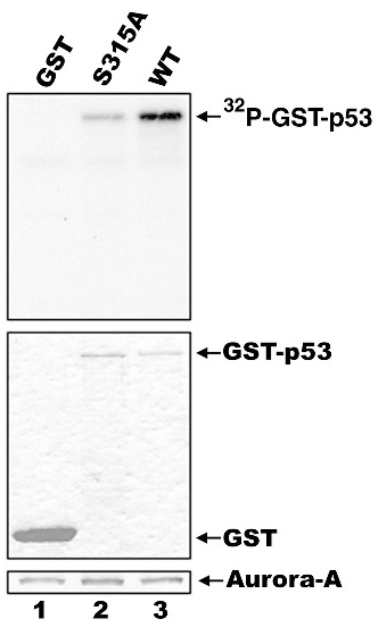

e
C
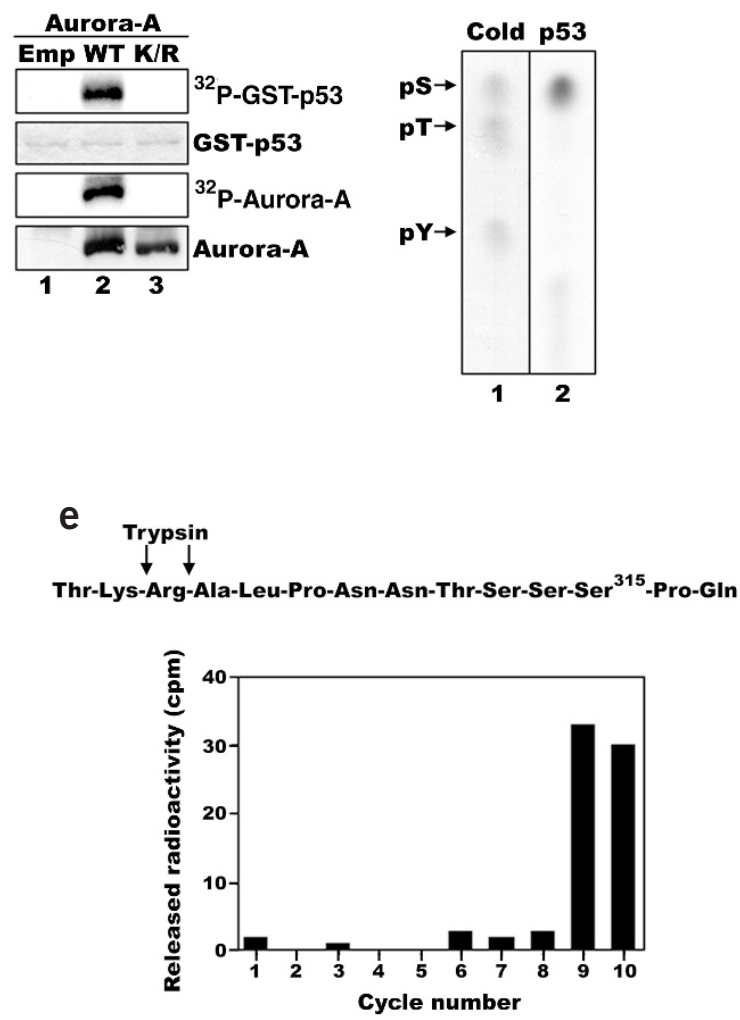

g

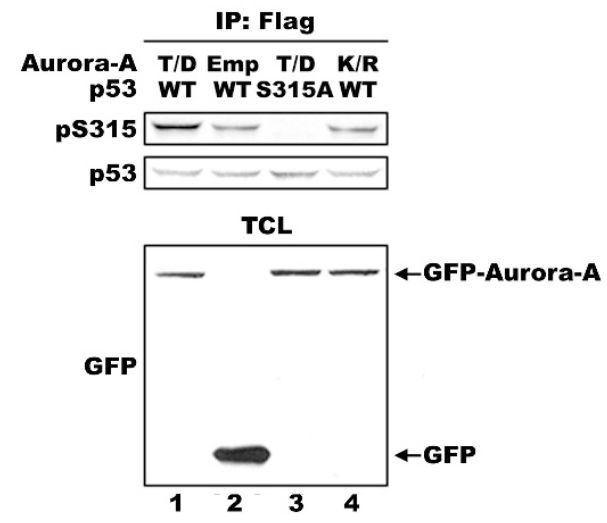

Figure 1 Aurora kinase A phosphorylates p53 at Ser315. (a) Immunoprecipitates with antibody to aurora kinase A from nocodazole-treated mitotic HeLa cells were incubated with either GST (lane 1) or GST-p53 (lane 2) in the presence of [ $\gamma^{32}$ P]ATP. GST proteins were resolved by SDS-PAGE and visualized by autoradiography (for kinase assay, top) or Coomassie blue staining (middle). Immunoprecipitates were detected with antibody to aurora kinase A (bottom). (b) Immunoprecipitates with antibody to Flag M2 from nocodazole-treated mitotic HeLa cells transfected with empty vector (Emp; lane 1), Flag-wild-type aurora kinase A (WT; lane 2) or Flag-aurora kinase A K162R (lane 3) were incubated with GST-p53 in the presence of [ $\gamma^{32}$ P]ATP. GST-p53 was resolved and visualized as in a (first and second rows). Immunoprecipitates were immunoblotted with antibody to Flag M2 (fourth row). Autophosphorylation of Flag-aurora kinase $A$ was visualized by autoradiography (third row). (c) Trypsin-digested and $\mathrm{HCl}$-treated ${ }^{32} \mathrm{P}$ labeled GST-p53 purified by SDS-PAGE was resolved by thin layer chromatography together with phosphorylated amino acid marker. Cold indicates the position of phosphorylated amino acid markers (pS, phosphoserine; $\mathrm{pT}$, phosphothreonine; pY, phosphotyrosine). (d) Trypsin-digested ${ }^{32} \mathrm{P}$-labeled peptides as in c were subjected to two-dimensional phosphopeptide mapping. Arrow indicates the major phosphopeptide. (e) Amino acid sequence including and surrounding Ser315 showing Nterminal trypsin cleavage sites (top). Phosphopeptide indicated by arrow was extracted from the thin-layer-chromatography plate and subjected to Edman degradation. Released radioactivity was monitored at each cycle (shown as bar graphs). (f) GST, GST-p53 S315A and GST-wild-type p53 (WT) were incubated with aurora kinase A immunoprecipitates and [ $\gamma^{32}$ P]ATP as in a. Proteins were resolved and visualized as in a. (g) Flag-wild-type p53 (WT; lanes 1, 2 and 4) or Flag-p53 S315A (lane 3) were cotransfected with GFP-aurora kinase A T288D (T/D; lanes 1 and 3), GFP-empty vector (Emp; lane 2) or GFP-aurora kinase A K162R (K/R; lane 4) into HEK293 cells. Twenty-four hours after transfection, p53 was immunoprecipitated (IP) with antibody to Flag M2 and immunoprecipitates were immunoblotted with antibody to p53 phosphorylated at Ser315 and antibody to p53 (top and middle). Aliquots of the same total cell lysate (TCL) were directly immunoblotted with antibody to GFP (bottom). 
the control GST alone. Aurora kinase A showed specific binding to p53 (Fig. 2a). We also carried out a reverse experiment in which we incubated GST-tagged p53 constructs representing the $\mathrm{N}$-terminal domain (amino acids 1-112), the central DNA binding domain (amino acids 93-290) and the C-terminal domain (amino acids 291-393) with ${ }^{35} \mathrm{~S}$ labeled in vitro-translated full-length aurora kinase A. Aurora kinase A bound to the C-terminal domain of p53 (residues 291-393; Fig. 2b). To map the aurora kinase A domains involved in this interaction, we tested the in vitro binding of GST-tagged deletion constructs of aurora kinase A with ${ }^{35}$ S-labeled in vitro-translated full-length p53. The Cterminal end of aurora kinase A (residues 312-403) showed no binding, but the rest of the aurora kinase A peptide, including the N-terminal end, did bind p53 (Supplementary Fig. 1 online). The aurora kinase A box, contrary to a previous report ${ }^{27}$, was not essential for this interaction.

We verified in vivo binding of endogenous aurora kinase A and p53 in Cos 1 cells by coimmunoprecipitation and immunoblotting experiments (Fig. 2c). We verified the specificity of this coimmunoprecipitation in p53-deficient Saos-2 cells, in which aurora kinase A did not precipitate with antibody to p53 (Supplementary Fig. 1 online). Coimmunoprecipitation experiments in MCF7 and HEK293 cells with wild-type p53 and in SW480 cells with mutant p53 (R273H) showed that aurora kinase A interacts with both wild-type and mutant p53 under physiological conditions in human cells (Fig. 2d). We evaluated the role of conformational changes to p53 in influencing the interaction between p53 and aurora kinase A in vivo in human cells with different p53 mutants. Coimmunoprecipitation experiments showed that p53 conformational mutants R175H and R249S had substantially less or no interaction with aurora kinase A (Supplementary Fig. 1 online), suggesting that conformation rather than functional inactivation is important for interaction of $\mathrm{p} 53$ with aurora kinase A. We carried out in vivo binding assays in p53-deficient $\mathrm{H} 1299$ cells cotransfected with wild-type or kinase-inactive aurora kinase A together with wild-type or S315A mutant $\mathrm{p} 53$. These assays showed that p53 could interact with both the wild-type and kinase-inactive forms of aurora kinase A with equal efficiency and that this interaction was not influenced by phosphorylation of p53 at Ser315 (Fig. 2e).

In vivo phosphorylation of p53 at Ser315 was previously mapped, but the biological relevance of this modification is controversial. Phosphorylation of p53 at Ser315 both enhances sequence-specific DNA-binding activity in vitro ${ }^{28}$ and reverses the stabilizing and activating effects of Ser392 phosphorylation on tetramer formation ${ }^{29}$. Because p53-mediated regulation of checkpoint response is activated when p53 is stabilized at both G1-S and G2-M transitions of the cell cycle $^{30}$, we investigated whether interaction between p53 and aurora kinase A and phosphorylation of p53 at Ser315 also varies in a cell cycle-dependent manner. In U2-OS cells, with wild-type p53, the steady-state level of aurora kinase A progressively increased from G1-S
Figure 2 Aurora kinase A interacts with p53. (a) ${ }^{35}$ S-labeled, in vitro-translated p53 was incubated with the beads bound either with GST or with GST-aurora kinase A. After binding, the beads were resolved by SDS-PAGE and visualized by autoradiography (for binding, top) or Coomassie blue staining (bottom). (b) ${ }^{35} \mathrm{~S}$-labeled, in vitro-translated aurora kinase A was incubated with the beads bound with GST, with GST-full length p53 (Full) or with a series of GST-p53 partial peptides ( $\mathrm{N}$-terminal ( $\mathrm{N}$-term), amino acids 1-112; DBD (DNA binding domain), amino acids 93-290; C-terminal (C-term), amino acids 291-393) followed by analysis as in a. (c) Cos 1 cells were immunoprecipitated (IP) with normal IgG (lane 1) or with antibody to aurora kinase A (lane 2). Immunoprecipitates were immunoblotted with antibody to p53 and antibody to aurora kinase A (left). Right panel shows reciprocal experiment. Immunoprecipitates with normal IgG (lane 3) or with antibody to p53 (lane 4) were immunoblotted with antibody to aurora kinase $A$ and antibody to p53. (d) MCF7 (lane 1), 293 (lane 2) and SW480 (lane 3 ) cells were immunoprecipitated (IP) with antibody to aurora kinase $\mathrm{A}$ and

immunoprecipitates were immunoblotted with indicated antibodies. (e) H1299 cells were either transfected with GFP-wild-type aurora kinase A (WT; lane 1), with GFP-aurora kinase A K162R (K/R; lane 2) or with GFP-wild-type aurora kinase A and wild-type p53 (lane 3), with GFP-aurora kinase A K162R and wild-type p53 (lane 4) or with GFP-wild-type aurora kinase A and p53 S315A (lane 5). Twenty-four hours after transfection, p53 was immunoprecipitated with antibody to p53 and immunoprecipitates were immunoblotted with indicated antibodies (top and middle). Aliquots of the same total cell lysates (TCL) were directly immunoblotted with antibody to GFP (bottom). Emp, empty vector. a
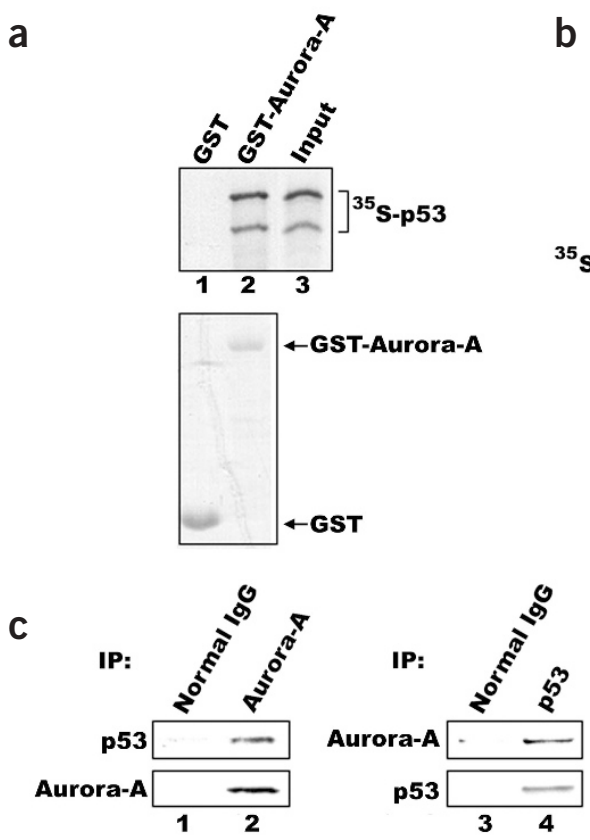

e

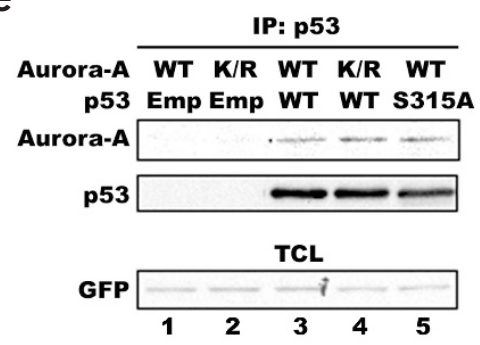

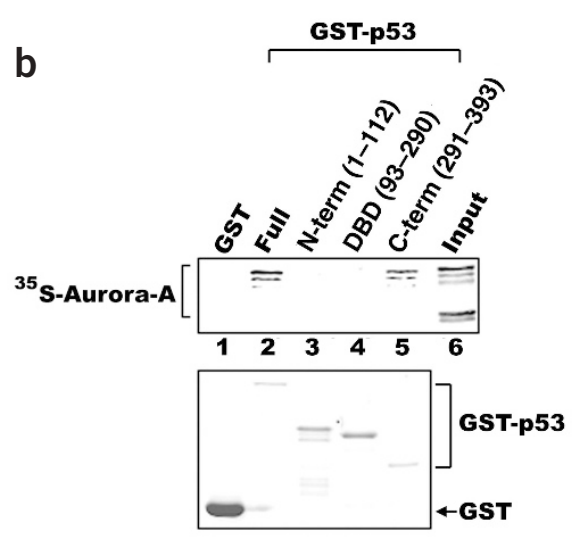

d

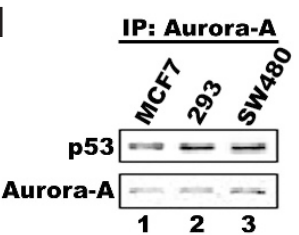


through G2-M phase, whereas the level of p53-bound aurora kinase A was comparable throughout the cell cycle. p53 phosphorylated at Ser315 was detectable throughout the cell cycle, but the relative amount of this species increased in S phase and during G2-M phase (Supplementary Fig. 2 online).

\section{Phosphorylation by aurora kinase A destabilizes p53}

Because gain of function of aurora kinase A through overexpression gives rise to cellular phenotypes similar to those seen with loss of function of $\mathrm{p} 53$, we hypothesized that phosphorylation of p53 at Ser 315 by aurora kinase A inactivates $\mathrm{p} 53$ by enhancing its proteolytic degradation. To address the issue, we cotransfected H1299 cells with increasing ratios of aurora kinase A to p53 in independent experiments. Increasing expression of aurora kinase A led to declining steady-state levels of p53, coinciding with less cyclin-dependent kinase inhibitor p21 (Fig. 3a). Overexpression of aurora kinase A did not have a similar effect on the level of endogenous and ectopically expressed p21 in the absence of p53 (Supplementary Fig. 3 online), suggesting that destabilization of $\mathrm{p} 53$ after overexpression of aurora kinase A affects downstream p53-transactivated effectors, such as p21. The levels of p27, also regulated by ubiquitin-proteasome degradation system $^{31}$, and a control GFP did not change in these experiments, indicating that aurora kinase A-mediated destabilization of p53 is not a nonspecific phenomenon. We assayed the effect of aurora kinase
Figure 3 Phosphorylation by aurora kinase $A$ destabilizes p53. (a) p53 or p27 was cotransfected with empty vector (Emp) and GFP (lane 1 ) or with different amounts of aurora kinase A and GFP into H1299 cells (lane 2-4). Twenty-four hours after transfection, aliquots of the same total cell lysates were immunoblotted with the indicated antibodies. Similar GFP expression indicates comparable transfection efficiency (bottom). (b) Wild-type p53 (WT; lanes 1-3) or p53 S315A (lane 4-6) was cotransfected with empty vector (Emp; lanes 1 and 4), with wild-type aurora kinase A (WT; lanes 2 and 5 ) or with aurora kinase A K162R (K/R; lanes 3 and 6) into H1299 cells and analyzed as in a. (c) Mdm2 was cotransfected with wild-type p53 (WT; lane 1), with p53 S315A (lane 2), with wild-type aurora kinase $A$ and wild-type p53 (lane 3 ) or with wild-type aurora kinase A and p53 S315A (lane 4) into $\mathrm{H} 1299$ cells, and cells were treated with protease inhibitor $\mathrm{N}$-acetyl-Leu-LeuNorleucinal for $6 \mathrm{~h}$ before harvest. Twenty-four hours after transfection, p53 was immunoprecipitated (IP) with antibody to p53 and then immunoblotted with antibody to p53. The same membrane was stripped and reprobed with indicated antibodies. pS315, p53 phosphorylated at Ser315. Short exposure shows the unubiquitinated single band of p53. Long exposure of the same membrane shows multiple ubiquitinated bands of p53 in addition to the single unubiquitinated band seen after short exposure. (d) p53 was cotransfected with empty vector (lane 1), with aurora kinase A (lane 2), with aurora kinase A and wild-type Mdm2 (WT; lane 3 ), with aurora kinase $A$ and a ubiquitinationdefective Mdm2 mutant (mt; lane 4) or with aurora kinase A, wild-type Mdm2 and mutant Mdm2 (lane 5) into H1299 cells and analyzed as in a. (e) p53 was cotransfected with wild-type Mdm2 (WT; lane 1), with aurora kinase $A$ and wild-type Mdm2 (lane 2), with aurora kinase A and mutant Mdm2 (mt; lane 3) or with aurora kinase A, wild-type Mdm2 and mutant Mdm2 (lane 4) into H1299 cells and analyzed as in c.

IP, immunoprecipitation. Short exposure shows the unubiquitinated single band of p53. Long exposure of the same membrane shows multiple ubiquitinated bands of p53 in addition to the single unubiquitinated band seen after short exposure. (f) Wild-type p53 (WT; lanes 1-3) or a p53 mutant that does not bind Mdm2 (mt; lanes 4-6) was cotransfected with empty vector (lanes 1 and 4), with aurora kinase A (lanes 2 and 5) or with aurora kinase A and wild-type Mdm2 (lanes 3 and 6) and analyzed as in a. (g) H1299 cells transfected with control siRNA duplex (GL2; lanes 1 and 2) or siRNA duplex targeting Mdm2 (lanes 3 and 4). Twenty-four hours after transfection, p53 and Mdm2 were cotransfected with either empty vector (lanes 1 and 3) or with aurora kinase A (lanes 2 and 4 ) for $24 \mathrm{~h}$ and cells were immunoblotted with indicated antibodies. p53* (second row) shows the normalized amount of p53 in the four samples. Normalization was done after densitometric analyses of p53 (third row) and GFP (fourth row) band intensities in each lane, using Scion Image and NIH Image software. (h) H1299 cells transfected with control siRNA duplex (GL2; lanes 1 and 2) or Mdm2 siRNA duplex (lanes 3 and 4 ) for 24 h were subjected to cotransfection with p53 (lanes 1 and 3) or with p53 and aurora kinase A together (lanes 2 and 4). After $24 \mathrm{~h}$, cells were analyzed as in a. 
A-mediated phosphorylation on the stability of $\mathrm{p} 53$ by expressing wild-type or S315A variants of p53 with wild-type or kinase-inactive mutants of aurora kinase A in H1299 cells. Only wild-type p53 was destabilized in the cells expressing wild-type aurora kinase A, suggesting that Ser315 phosphorylation by aurora kinase A facilitates degradation of p53 (Fig. 3b).

Because degradation of p53 predominantly involves Mdm2-mediated ubiquitination, we assayed ubiquitination of p53 in H1299 cells cotransfected with $\mathrm{Mdm} 2$ and either empty vector or vector expressing aurora kinase $\mathrm{A}$ in combination with wild-type or S315A mutant p53. In cells expressing wild-type p53, we observed substantially more ubiquitination in the presence of aurora kinase A than in presence of the empty vector. We observed moderate ubiquitination in cells transfected with the empty vector, probably as a result of phosphorylation of p53 by endogenous aurora kinase A. We observed less ubiquitination of p53 in cells expressing the S315A mutant p53 (Fig. 3c). The difference in ubiquitination

Figure 4 Effect of aurora kinase $A$ expression on p53 stability and function in vivo. (a) Lysates from U2-OS cells transfected with control siRNA duplex (GL2; lane 1) or siRNA duplex targeting aurora kinase $\mathrm{A}$ (lane 2) for $48 \mathrm{~h}$ were immunoblotted with indicated antibodies. The same lysates were immunoprecipitated with antibody to p53 and immunoblotted with the indicated antibodies. The relative ratio of $p 53$ phosphorylated at Ser315 (pS315) to total immunoprecipitated p53 was then estimated.

(b) Cell cycle in a was analyzed by FACS.

(c) Lysates from MCF7 cells transfected with control siRNA duplex (GL2; lane 1) or siRNA duplex targeting aurora kinase A (lane 2) for $72 \mathrm{~h}$ were analyzed as in a. (d) Lysates from MCF7 cells transfected with siRNA for $72 \mathrm{~h}$ as in $\mathbf{c}$ were collected at the indicated time points after addition of cycloheximide ( $\mathrm{CHX}$ ) and subjected to immunoblotting with the indicated antibodies.

The amount of p53 was quantified by

densitometry and is shown relative to the amount of p53 expressed in absence of cycloheximide. (e) Lysates from a stable clone expressing vector (lane 1), a stable clone expressing aurora kinase A (lane 2) and a stable clone expressing aurora kinase A treated with siRNA duplex (lane 3) were analyzed as in a. (f) Cells in e were treated with cycloheximide $(\mathrm{CHX})$ and analyzed as in $\mathbf{d}$.

(g) MCF7 cells transfected with siRNA duplex for $48 \mathrm{~h}$ were treated with the indicated concentrations of cisplatin, and $24 \mathrm{~h}$ later the number of apoptotic cells was counted. (h) MCF7 cells were transfected with vector and GFP together or aurora kinase A and GFP together for $48 \mathrm{~h}$, then treated with cisplatin and analyzed as in g. (i) Stable clones expressing vector and aurora kinase A were treated with cisplatin for 24 $\mathrm{h}$ and the sub-G1 cell population was analyzed by FACS. Expression levels of p53 in the same cells was analyzed as in $\mathbf{a}$. The amount of p53 in the control and the cisplatin-treated cells was quantified to assess the relative p53 expression in the cisplatin-treated cells.

i was not due to differences in amount of p53, as comparable levels of p53 were immunoprecipitated from all four transfections. We also noted greater binding of $\mathrm{Mdm} 2$ to highly ubiquitinated p53 in cells expressing aurora kinase A (Fig. 3c). Consistent with the levels of ubiquitinated p53, bound Mdm2 was more abundant in the cells transfected with empty vector and expressing wild-type p53 than in those expressing S315A mutant p53, possibly owing to phosphorylation of wild-type $\mathrm{p} 53$ by endogenous aurora kinase $\mathrm{A}$.
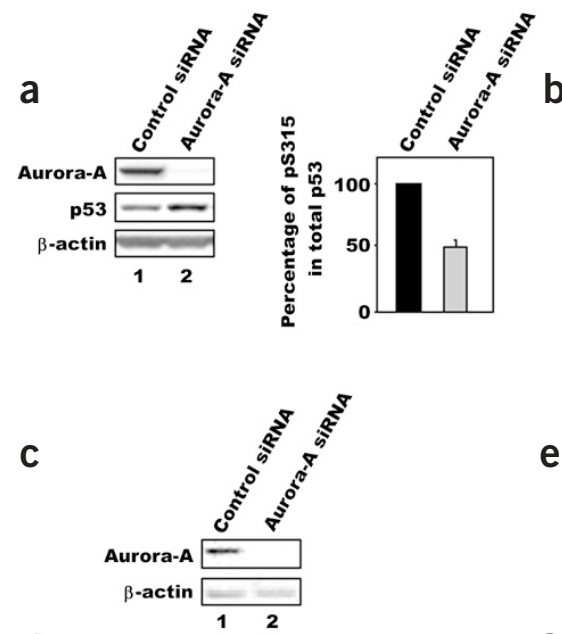

d
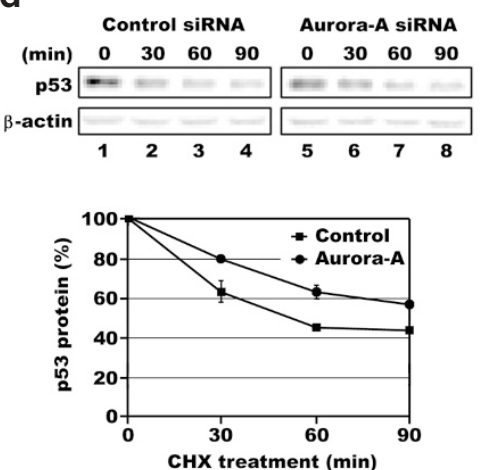

g

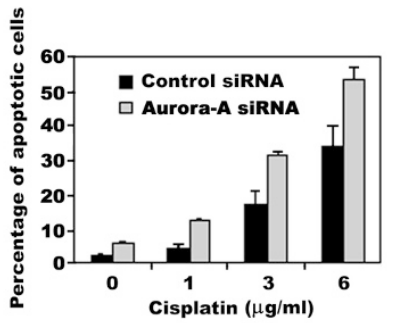

h

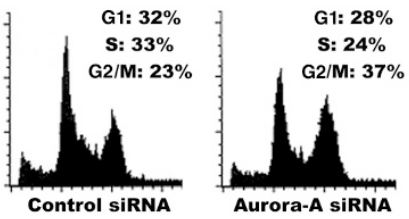

e

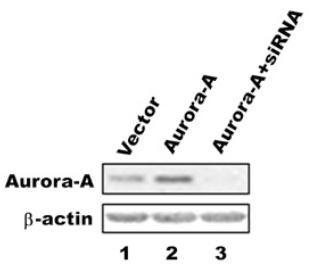

f
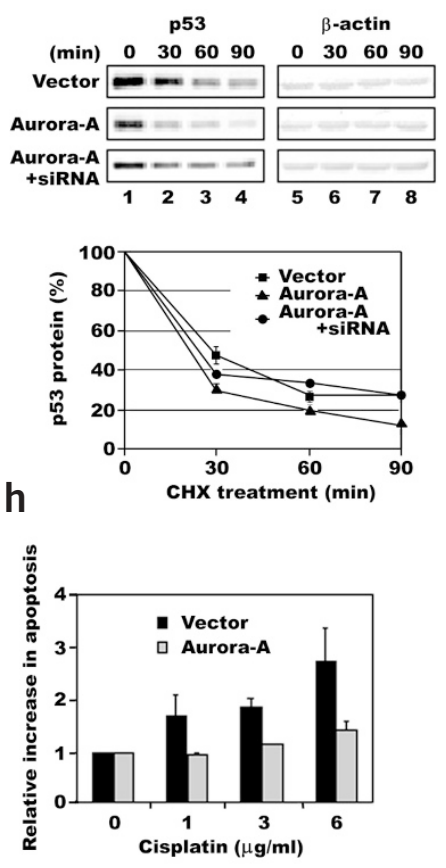
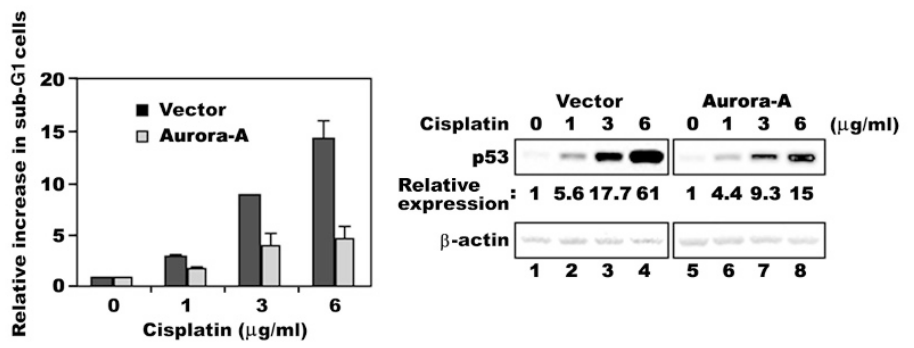

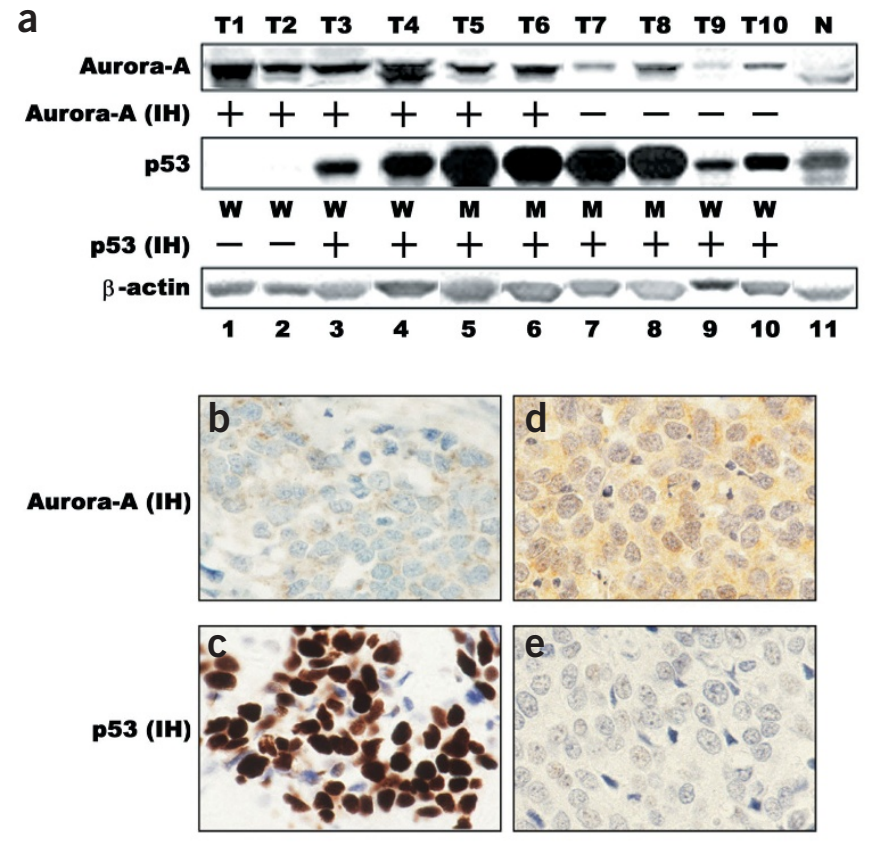

To further investigate the role of Mdm2 in destabilization of p53 phosphorylated by aurora kinase A, we cotransfected H1299 cells with either mutant Mdm2 (C464A) lacking ubiquitination activity ${ }^{32}$ or wild-type Mdm2 and aurora kinase A. The level of p53 was low in cells transfected with wild-type Mdm2 but unaltered in cells transfected with mutant Mdm2 compared with control cells not transfected with Mdm2 (Fig. 3d). Mutant Mdm2 also had a dominant negative effect of stabilizing p53 in the presence of aurora kinase A.

We further documented the role of aurora kinase A in Mdm2-mediated ubiquitination of $\mathrm{p} 53$ using ubiquitination assays on cells transfected with the same combinations of expression constructs described above (Fig. 3e). Ubiquitinated p53 in cells expressing aurora kinase A, p53 and wild-type Mdm2 was much more abundant than in cells expressing mutant Mdm2. As expected, ubiquitination of $\mathrm{p} 53$ was minimal in the absence of aurora kinase A and was undetectable in cells expressing mutant Mdm2. Mutant p53 (L22Q and W23S) that cannot interact with Mdm2 (ref. 33) was also not destabilized when expressed with aurora kinase $\mathrm{A}$ and $\mathrm{Mdm} 2$ (Fig. 3f). Silencing of $\mathrm{Mdm} 2$ with small interfering RNA (siRNA) in cells with transfected (Fig. 3g) and endogenous (Fig. 3h) Mdm2 inhibited destabilization of p53 in the presence of aurora kinase A. Absence of a perceptible increase in the amount of p53 after silencing of Mdm2 by siRNA (Fig. $3 \mathbf{h}$ ), which seems to conflict with the existing model of $\mathrm{p} 53$ regulation, may reflect the fact that proliferating cells can tolerate only limited amount of p53. These results, taken together, indicate that Mdm2-mediated ubiquitination has a central role in the destabilization of p53 induced by aurora kinase A.

\section{Phosphorylation of p53 at Ser315 affects cell cycle}

Because p53 is a crucial regulator of cell cycle progression and DNA damage-induced checkpoint response in mammalian cells ${ }^{30}$, we investigated whether inhibition of physiological levels of aurora kinase A with siRNA affects stability of p53 and, consequently, p53-regulated pathways in U2-OS and MCF7 cells with wild-type p53. Treatment of $\mathrm{U} 2-\mathrm{OS}$ cells for $48 \mathrm{~h}$ with aurora kinase A siRNA resulted in substantial depletion of aurora kinase A, which was directly correlated with an increase in the steady-state level of p53 and inversely correlated with the amount of p53 phosphorylated at Ser315 (Fig. 4a). These cells also
Figure 5 Steady-state level of aurora kinase A and p53 in human bladder tumor samples. (a) Expression levels of aurora kinase A (top), p53 (middle) and $\beta$-actin (bottom) in bladder tumor tissues (T1-T10; lanes 1-10) and in a normal bladder epithelial cells ( $\mathrm{N}$; lane 11 ). Intensity of immunohistochemical $(\mathrm{IH})$ staining of each tumor specimen compared with normal tissue is represented as follows: -, no detectable expression or similar expression; +, higher expression. W, wild-type p53; M, mutant p53. (b-e) Immunohistochemical (IH) staining of tumor specimens with antibody to aurora kinase $\mathrm{A}(\mathbf{b}, \mathbf{d})$ and antibody to p53 (c,e). b and $\mathbf{c}$ represent the same sample, and $\mathbf{d}$ and $\mathbf{e}$ represent the same sample.

had a larger G2-M population of $37 \%$, compared with $23 \%$ in cells treated with control siRNA (Fig. 4 b). This growth arrest or delay in the G2-M phase after depletion of aurora kinase A could be due to stabilization of p53. The lack of a similar growth-inhibitory effect in p53deficient H1299 and Saos-2 cells depleted of aurora kinase A (data not shown) supports this conclusion.

We then assessed the stability of p53 in MCF7 parental cells and in MCF7 cells stably transfected with aurora kinase A with and without silencing of aurora kinase A by exposure to cycloheximide, a translational inhibitor, in time-course experiments. Treatment with aurora kinase A siRNA for $72 \mathrm{~h}$ almost completely inhibited expression of aurora kinase A (Fig. 4c,e). We estimated the relative amount of p53 in cells exposed to cycloheximide for 30, 60 and $90 \mathrm{~min}$ and found consistently higher levels of p53 in cells treated with aurora kinase A siRNA than in cells treated with a control siRNA. Treatment with cycloheximide for 90 min decreased p53 level by almost $60 \%$ in the control cells but only by $\sim 40 \%$ in cells with silenced aurora kinase A (Fig. 4d), indicating that the degradation rate of p53 was greater in cells expressing aurora kinase A. We observed a similar phenomenon in cells overexpressing aurora kinase A: silencing of aurora kinase A in these cells stabilized p53 to almost the same extent as seen in control cells (Fig. 4f). These results suggest that expression of aurora kinase A at physiological levels in vivo mediates degradation of $\mathrm{p} 53$ and that this effect is enhanced in cells overexpressing aurora kinase A, possibly causing abrogation of p53-mediated checkpoint response pathways.

\section{Aurora kinase A affects p53-mediated apoptosis}

We investigated whether aurora kinase A affects p53-induced apoptosis in response to cisplatin-induced DNA damage in MCF7 cells. Silencing of aurora kinase A with siRNA substantially increased the incidence of apoptosis in these cells. Treatment with $6 \mu \mathrm{g} \mathrm{ml}{ }^{-1}$ cisplatin increased the incidence of apoptosis by $\sim 20 \%$ in cells with silenced aurora kinase A over that observed in cells transfected with a control siRNA (Fig. 4g). The lack of a similar increase in apoptotic response in p53-deficient H1299 cells treated with cisplatin (data not shown) indicated that the apoptotic response in MCF7 cells treated with cisplatin was a reflection of the effect of aurora kinase A on stability of p53. Consistent with its role as an inhibitor of $\mathrm{p} 53$ function, overexpression of aurora kinase A in transiently transfected cells inhibited p53-induced apoptotic response after treatment with cisplatin (Fig. $4 \mathbf{h}$ ). Based on the number of sub-G1 DNA-harboring cells generated, cells stably transfected with aurora kinase A were also resistant to cisplatin-induced apoptosis. Substantially less induction of p53 in these cells corroborated the role of aurora kinase $\mathrm{A}$ as an inhibitor of $\mathrm{p} 53$-induced apoptotic response in DNA-damaged cells (Fig. 4i). The apoptotic response was not mediated through the two p53 homologous family members p63 and p73, as cotransfection with increasing amounts of aurora kinase A had no effect on the stability of p63 $\gamma$ and $\mathrm{p} 73 \alpha$ isoforms, implicated in $\mathrm{p} 53$ like functions, in untreated (Supplementary Fig. 3 online) and cisplatintreated cells (data not shown). 


\section{Overexpression of aurora kinase A and low p53 levels}

In agreement with the experimental data, we detected a trend between overexpression of aurora kinase A and reduced expression of p53 in human bladder tumors (Fig. 5), which frequently overexpress aurora kinase $\mathrm{A}$, according to a recent report ${ }^{13}$. Of 23 tumors analyzed, 18 had no mutations of $\mathrm{p} 53$. Of these 18,15 showed high expression of aurora kinase $\mathrm{A}$, and 10 of these had reduced expression of $\mathrm{p} 53$.

\section{DISCUSSION}

Our findings indicate that aurora kinase A interacts with and phosphorylates 53 at Ser315, facilitating Mdm2-mediated ubiquitination and destabilization of $\mathrm{p} 53$. In view of this finding and the observed growth arrest of cells at G2-M phase after silencing of aurora kinase A, it is logical to suggest that degradation of p53 phosphorylated at Ser315 has physiological relevance related to allowing progression of cells through the normal cell proliferation cycle. Identification of aurora kinase $\mathrm{A}$ as an in vivo kinase for Ser315 does not rule out possible involvement of other S- and G2-phase cyclin-dependent kinases in the process, as precedents for multiple distinct kinases targeting the same phosphorylation site of p53 (ATM and ATR for Ser15; CK2 and PKR for Ser392) have been reported ${ }^{34-36}$.

Phosphorylation of Ser315 has been implicated in enhancing sequence-specific DNA-binding affinity and transactivation function of p53. Total phosphorylation profile, rather than phosphorylation of a single residue, may determine the functional status of p53 at any given stage of the cell cycle. Indeed, the fact that phosphorylation of Ser315 along with Ser33 and Thr81 leads to binding of p53 to prolyl isomerase Pin 1 (refs. 37,38), which stimulates transactivation function and stabilization of p53, indicates that phosphorylation of Ser315 can direct the functional status of p53 to either stabilization or degradation. Selectivity towards a particular response may depend on the total profile of post-translational modifications on p53 and the resultant recruitment of additional proteins required for such a response.

The mechanism by which phosphorylation by aurora kinase A enhances Mdm2-mediated ubiquitination of p53 is not known at this time. Greater binding of $\mathrm{Mdm} 2$ to phosphorylated p53 undergoing ubiquitination suggests that p53 phosphorylated by aurora kinase A has greater binding affinity for Mdm2. Ser315 phosphorylation may help oligomerization of $\mathrm{p} 53$, which is required for Mdm2-mediated ubiquitination $^{39}$. The ability of Mdm2 to degrade p53 requires shuttling between the nucleus and the cytoplasm ${ }^{40,41}$, and inhibition of p53 nuclear export leads to accumulation of ubiquitinated p53 in the nucleus ${ }^{32}$. Localization of aurora kinase A in both nucleus and cytoplasm ${ }^{42-44}$ makes it a credible catalyst for p53 ubiquitination.

A recent study using an ectopically expressed transactivation- and oligomerization-defective p53 deletion mutant found that oncogenic activity of aurora kinase A is suppressed by p53 in a transactivationindependent manner ${ }^{27}$. This observation, however, is inconsistent with our model of aurora kinase A function. Because the study cited used a transactivation- and oligomerization-defective p53 deletion mutant for functional assays, the physiological relevance of the reported finding needs to be investigated further.

The most important tumor-suppressor function of p53 involves its ability to induce apoptosis; thus, a negative regulator of p53, such as aurora kinase $\mathrm{A}$, must have a crucial role in tumorigenic transformation of cells. This function of aurora kinase A may explain why overexpression causes oncogenic transformation in mammalian cells and why amplification or overexpression of aurora kinase A is commonly detected in human cancers ${ }^{8-13}$. Notably, our findings also provide a mechanistic explanation for the recently reported role of aurora kinase $\mathrm{A}$ as a tumor-susceptibility protein in mouse and human ${ }^{45}$.
Overexpression of aurora kinase A resulting in loss of p53 function confers resistance to DNA damage-inducing agents, such as cisplatin, a drug commonly used in cancer chemotherapy. It is, therefore, expected that overexpression of aurora kinase A would be under positive selection in human tumors expressing wild-type $\mathrm{p} 53$. The function of aurora kinase A as a p53-destabilizing molecule makes it an important target for developing therapeutic strategies for those human cancers in which aurora kinase $\mathrm{A}$ is overexpressed.

\section{METHODS}

Mapping of phosphorylation site. We produced GST-aurora kinase A and GST-p53 proteins in BL21 pLys bacteria according to the manufacturer's protocol (Amersham Pharmacia Biotech). We carried out in vitro kinase assays as described ${ }^{14}$. We purified ${ }^{32} \mathrm{P}$-labeled GST-p 53 phosphorylated by aurora kinase $\mathrm{A}$ in vitro by SDS-PAGE and digested it with N-tosyl-L-pheylalanine chloromethyl ketone-trypsin (Sigma). We subjected digested phosphopeptides to phosphorylated amino acid analysis and two-dimensional analysis using the HTLE-7000 electrophoresis system (CBS Scientific) as described ${ }^{25}$. We carried out manual Edman degradation as described ${ }^{26}$.

In vitro binding assay. We produced in vitro-translated proteins in the presence of ${ }^{35} \mathrm{~S}$-methionine using the TNT coupled transcription/translation kit (Promega). We incubated GST-aurora kinase A or GST-p53 fusion proteins bound to glutathione-Sepharose beads with ${ }^{35} \mathrm{~S}$-labeled $\mathrm{p} 53$ or aurora kinase A, respectively, in binding buffer (20 mM Tris (pH 7.4), $100 \mathrm{mM} \mathrm{NaCl}, 1 \mathrm{mM}$ EDTA, $1 \mathrm{mM}$ dithiothreitol, $0.1 \%$ Nonidet P-40) for $1 \mathrm{~h}$ at $4{ }^{\circ} \mathrm{C}$ and then washed them five times in wash buffer $(20 \mathrm{mM}$ Tris ( $\mathrm{pH} 7.4), 100 \mathrm{mM} \mathrm{NaCl}$, $1 \mathrm{mM}$ EDTA, $1 \mathrm{mM}$ dithiothreitol, $0.25 \%$ Nonidet P-40, 10\% glycerol). We analyzed the bound proteins by SDS-PAGE.

In vivo binding assay. We transfected cells using LipofectAMINE according to the manufacturer's protocol (Invitrogen). We extracted cells with lysis buffer (50 mM Tris ( $\mathrm{pH} \mathrm{8.0),} 20 \mathrm{mM}$ sodium glycerophosphate, $140 \mathrm{mM} \mathrm{NaCl}$, $25 \mathrm{mM} \mathrm{NaF}, 1 \mathrm{mM}$ phenylmethylsulfonyl fluoride, $1 \%$ Nonidet P-40, $0.5 \mu \mathrm{M}$ okadaic acid, $10 \mu \mathrm{M}$ Microcystin LR, Protease inhibitor cocktail) $24 \mathrm{~h}$ after transfection. We incubated the lysate for $20 \mathrm{~min}$ on ice and then centrifuged it at $15,000 \mathrm{~g}$ for $20 \mathrm{~min}$. We used the supernatant for immunoblotting and immunoprecipitation. For immunoprecipitation, we incubated $0.3-2 \mathrm{mg}$ of lysate for $3 \mathrm{~h}$ at $4{ }^{\circ} \mathrm{C}$ with $15 \mu \mathrm{l}$ of polyclonal antibody to aurora kinase $\mathrm{A}$ conjugated to protein G-agarose or $5 \mu \mathrm{g}$ of monoclonal antibody to p53 (Ab-6, Oncogene) conjugated to protein G-agarose, washed the immunocomplex four times with lysis buffer and then subjected it to SDS-PAGE.

p53 degradation and ubiquitination assay. We transfected p53-deficient H1299 cells with increasing amounts of either pcDNA3-Flag-tagged aurora kinase A or K162R mutant aurora kinase A together with $0.4 \mu \mathrm{g}$ of pcDNA3-Flag-tagged p53, S315A mutant p53 or L22Q/W23S double mutant p53; pCMV-Mdm2 or C464A mutant Mdm2; pCEP4-p27, pcDNA3-empty vector and pEGFP-empty vector in separate transfections. We detected levels of p53, p21, p27 and GFP by immunoblotting with the respective antibodies. For this purpose, we used antibody to p21 F-5 (Santa Cruz), antibody to p27 F-8 (Santa Cruz) and antibody to GFP Living Colors A.v. Peptide (Clontech). To evaluate ubiquitination of p53, we treated transfected H1299 cells for $6 \mathrm{~h}$ with a proteasome inhibitor LLnL (50 $\mu \mathrm{M}$; Sigma), collected them and extracted them with lysis buffer. We immunoprecipitated the lysate with monoclonal antibody to p53 (Ab-6) and then resolved it by SDS-PAGE ( $8 \%$ gel) and analyzed it by immunoblotting with antibody to p53 (Ab-6), antibody to p53 phosphorylated at Ser315 and monoclonal antibody to Mdm2 (Ab-1, Oncogene Science). To assess stability of p53, we treated MCF7 cells with $80 \mu \mathrm{g} \mathrm{ml}^{-1}$ of cycloheximide for indicated times.

siRNA, stable clone and cisplatin treatment. We carried out siRNA experiments for aurora kinase A and $\mathrm{Mdm} 2$ as previously described ${ }^{46,47}$. We obtained aurora kinase A stable transfectants by transfecting pcDNA3-wild-type aurora kinase A to MCF7 cells and then selecting the cells in the presence of G418 for 3 weeks. We added cisplatin to the culture medium for $24 \mathrm{~h}$ at indicated concentrations. For apoptosis analysis of siRNA-transfected cells, we fixed the cells in $3.7 \%$ formaldehyde, stained them with DAPI and counted the nuclear morphology of at least 
500 cells under the fluorescence microscope. For FACS analysis of siRNA-transfected cells and stable transfectants, we stained the cells with propidium iodide and analyzed them by Becton Dickinson FACSCAN flow cytometer ${ }^{48}$.

Bladder tissue samples. We analyzed the expression levels of aurora kinase A kinase and p53 in 23 bladder tumor tissues and 2 normal tissues by immunoblotting and immunohistochemistry as described ${ }^{13}$. We obtained frozen tissue samples from the interdepartmental tissue repository at the University of Texas M.D. Anderson Cancer Center. The tissue samples were collected under the approved institutional laboratory protocols and all individuals from whom samples were collected signed the informed. consent. We isolated exon 5-9 of TRP53 (encoding p53) from all tumor samples, amplified the region and directly sequenced it by applying the cycle sequencing dye terminator protocol (PE Applied Biosystems).

Note: Supplementary information is available on the Nature Genetics website.

\section{ACKNOWLEDGMENTS}

We thank B. Vogelstein and C. Lengauer for Mdm2, p21 and p27 expression constructs; T. Hupp and E. Appella for antibodies specific to $\mathrm{p} 53$ phosphorylated at Ser315; J. Dai for BT549 cells; H. Grossman for normal human uroepithelial cells; J. Liu for help with the two-dimensional protein gel and analysis of phosphorylated amino acids; and G. Lozano, J. Barboza and J. Carter for helpful discussion and critical reading of the manuscript. This work was supported by grants from the US National Institutes of Health, US Department of Defense Breast Cancer Research Program, and The University of Texas M.D. Anderson Cancer Center Human Cancer Genetics Development Funds (to S.S.). DNA sequencing was done at the M.D. Anderson Cancer Center DNA sequencing facility, supported by a US National Institutes of Health Core grant.

\section{COMPETING INTERESTS STATEMENT}

The authors declare that they have no competing financial interests.

Received 5 October; accepted 12 November 2003

Published online at http://www.nature.com/naturegenetics/

1. Nigg, E.A. Mitotic kinases as regulators of cell division and its checkpoints. Nat. Rev Mol. Cell. Biol. 2, 21-32 (2001).

2. Glover, D.M., Leibowitz, M.H., McLean, D.A. \& Parry, H. Mutations in aurora prevent centrosome separation leading to the formation of monopolar spindles. Cell $\mathbf{8 1}$, 95-105 (1995).

3. Chan, C.S. \& Botstein, D. Isolation and characterization of chromosome-gain and increase-in-ploidy mutants in yeast. Genetics 135, 677-691 (1993).

4. Bischoff, J.R. \& Plowman, G.D. The Aurora/Ipl1p kinase family: regulators of chromosome segregation and cytokinesis. Trends Cell Biol. 9, 454-459 (1999).

5. Giet, R. \& Prigent, C. Aurora/Ipl1p-related kinases, a new oncogenic family of mitotic serine-threonine kinases. J. Cell Sci. 112, 3591-3601 (1999).

6. Goepfert, T.M. \& Brinkley, B.R. The centrosome-associated Aurora/lpl-like kinase family. Curr. Top. Dev. Biol. 49, 331-342 (2000).

7. Hirota, T. et al. Aurora-A and an interacting activator, the LIM protein Ajuba, are required for mitotic commitment in human cells. Cell 114, 585-598 (2003).

8. Zhou, H. et al. Tumour amplified kinase STK15/BTAK induces centrosome amplification, aneuploidy and transformation. Nat. Genet. 20, 189-193 (1998).

9. Bischoff, J.R. et al. A homologue of Drosophila aurora kinase is oncogenic and amplified in human colorectal cancers. EMBO J. 17, 3052-3065 (1998).

10. Sen, S., Zhou, H. \& White, R.A. A putative serine/threonine kinase encoding gene BTAK on chromosome $20 q 13$ is amplified and overexpressed in human breast cancer cell lines. Oncogene 14, 2195-2200 (1997).

11. Tanaka, T. et al. Centrosomal kinase AIK1 is overexpressed in invasive ductal carcinoma of the breast. Cancer Res. 59, 2041-2044 (1999).

12. Miyoshi, Y., Iwao, K., Egawa, C. \& Noguichi, S. Association of centrosomal kinase STK15/BTAK mRNA expression with chromosomal instability in human breast cancers. Int. J. Cancer 92, 370-373 (2001).

13. Sen, S. et al. Amplification/overexpression of a mitotic kinase gene in human bladder cancer. J. Natl. Cancer Inst. 94, 1320-1329 (2002).

14. Katayama, H., Zhou, H., Li, Q., Tatsuka, M. \& Sen, S. Interaction and feedback regulation between STK15/BTAK/aurora-A kinase and protein phosphatase 1 through mitotic cell division cycle. J. Biol. Chem. 276, 46219-46224 (2001).

15. Fukasawa, K., Choi, T., Kuriyama, R., Rulong, S. \& VandeWonde, G.F. Abnormal centrosome amplification in the absence of p53. Science 271, 1744-1747 (1996)

16. Bischoff, F.Z. et al. Spontaneous abnormalities in normal fibroblasts from patients with Li-Fraumeni cancer syndrome: aneuploidy and immortalization. Cancer Res. 50, 7979-7984 (1990).

17. Kubbutat, M.H., Jones, S.N. \& Vousden K.H. Regulation of p53 stability by Mdm2. Nature 387, 299-303 (1997).

18. Shieh, S.Y., Ikeda, M., Taya, Y. \& Prives, C. DNA damage-induced phosphorylation of p53 alleviates inhibition by Mdm2. Cell 91, 325-334 (1997).

19. Shieh, S.Y., Taya, Y. \& Prives, C. DNA damage-inducible phosphorylation of p53 at Nterminal sites including a novel site, Ser20, requires tetramerization. EMBO J. 18, 1815-1823 (1999).

20. Shieh, S.Y., Ahn, J., Tamai, K., Taya, Y. \& Prives, C. The human homologs of checkpoint kinases Chk1 and Cds1 (Chk2) phosphorylate p53 at multiple DNA damageinducible sites. Genes Dev. 14, 289-300 (2000).

21. Sakaguchi, K. et al. DNA damage activates p53 through a phosphorylation-acetylation cascade. Genes Dev. 12, 2831-2841 (1998).

22. Shaw, P., Freeman, J., Bovey, R. \& Iggo, R. Regulation of specific DNA binding by p53: evidence for a role for 0 -glycosylation and charged residues at the carboxy-terminus. Oncogene 12, 921-930 (1996).

23. Rodriguez, M. S., Dargemont, C. \& Hay, R.T. SUMO-1 conjugation in vivo requires both a consensus modification motif and nuclear targeting. J. Biol. Chem. 276, 12654-12659 (2001).

24. Bech-Otschir, D. et al. COP9 signalosome-specific phosphorylation targets p53 to degradation by the ubiquitin system. EMBO J. 20, 1630-1639 (2001).

25. Liu, J. et al. Inhibition of Bcr serine kinase by tyrosine phosphorylation. Mol. Cell. Biol.16, 998-1005 (1996)

26. Zhou, B.P. et al. Cytoplasmic localization of p21Cip1/WAF1 by Akt-induced phosphorylation in HER-2/neu-overexpressing cells. Nat. Cell Biol. 3, 245-252 (2001).

27. Chen, S.S., Chang, P.C., Cheng, Y.W., Tang, F.M.\& Lin, Y.S. Suppression of the STK15 oncogenic activity requires a transactivation-independent p53 function. EMBO J. 21 4491-4499 (2002).

28. Wang, Y. \& Prives, C. Increased and altered DNA binding of human p53 by S and G2/M but not G1 cyclin-dependent kinases. Nature 376, 88-91 (1995).

29. Sakaguchi, K. et al. Phosphorylation of serine 392 stabilizes the tetramer formation of tumor suppressor protein p53. Biochemistry 36, 10117-10124 (1997).

30. Vogelstein, B., Lane, D. \& Levine, A.J. Surfing the p53 network. Nature 408, 307-310 (2000).

31. Boehm, M. et al. A growth factor-dependent nuclear kinase phosphorylates p27(Kip1) and regulates cell cycle progression. EMBO J. 21, 3390-3401 (2002).

32. Geyer, R.K., Yu, Z.K. \& Maki, C.G. The Mdm2 RING-finger domain is required to promote p53 nuclear export. Nat. Cell Biol. 2, 569-573 (2000)

33. Lin, J., Chen, J., Elenbaas, B. \& Levine, A.J. Several hydrophobic amino acids in the p53 amino-terminal domain are required for transcriptional activation, binding to mdm-2 and the adenovirus 5 E1B 55-kD protein. Genes Dev. 8, 1235-1246 (1994).

34. Banin, S. et al. Enhanced phosphorylation of p53 by ATM in response to DNA damage. Science 281, 1674-1677 (1998).

35. Tibbetts, R.S. et al. A role for ATR in the DNA damage-induced phosphorylation of p53. Genes Dev. 13, 152-157 (1999).

36. Cuddihy, A.R., Wong, A.H., Tam, N.W., Li, S. \& Koromilas, A.E. The double-stranded RNA activated protein kinase PKR physically associates with the tumor suppressor p53 protein and phosphorylates human p53 on serine 392 in vitro. Oncogene 18, 2690-2702 (1999).

37. Zheng, H. et al. The prolyl isomerase Pin 1 is a regulator of $\mathrm{p} 53$ in genotoxic response. Nature 419, 849-853 (2002).

38. Zacchi, P. et al. The prolyl isomerase Pin 1 reveals a mechanism to control p53 functions after genotoxic insults. Nature 419, 853-857 (2002).

39. Maki, C.G. Oligomerization is required for p53 to be efficiently ubiquitinated by Mdm2. J. Biol. Chem. 274, 16531-16535 (1999).

40. Woods, D.B. \& Vousden, K.H. Regulation of p53 function. Exp. Cell. Res. 264, 56-66 (2001).

41. Momand, J., Wu, H.H. \& Dasgupta, G. Mdm2-master regulator of the p53 tumor suppressor protein. Gene 242, 15-29 (2000).

42. Kiat, L.S., Hui, K.M. \& Gopalan, G. aurora-A kinase interacting protein (AIP), a novel negative regulator of human aurora-A kinase. J. Biol. Chem. 277, 45558-45565 (2002).

43. Crosio, C. et al. Mitotic phosphorylation of histone H3: spatio-temporal regulation by mammalian Aurora kinases. Mol. Cell. Biol. 22, 874-885 (2002).

44. Stenoien, D.L., Sen, S., Mancini, M.A. \& Brinkley, B.R. Dynamic association of a tumor amplified kinase, Aurora-A, with the centrosome and mitotic spindle. Cell Motil. Cytoskeleton 55, 134-146 (2003).

45. Ewart-Toland, A. et al. Identification of Stk6/STK15 as a candidate low-penetrance tumor-susceptibility gene in mouse and human. Nat. Genet. 34, 403-412 (2003).

46. Kufer, T.A. et al. Human TPX2 is required for targeting Aurora-A kinase to the spindle. J. Cell Biol. 158, 617-623 (2002).

47. Brès, V. et al. A non-proteolytic role for ubiquitin in Tat-mediated transactivation of the HIV-1 promoter. Nature Cell Biol. 8, 754-761 (2003).

48. Sen, S. et al. Expression of a gene encoding a tRNA synthetase like protein is enhanced in tumorigenic human myeloid leukemia cells and is cell cycle stage and differentiation dependent. Proc. Natl. Acad. Sci. USA 94, 6164-6169 (1997). 